\title{
An Exploratory Study on the Personal Qualities/Characteristics expected by the Organisations for Key HRM jobs in Sri Lanka
}

\author{
A. Anton Arulrajah \\ Senior Lecturer \\ Department of Management \\ Faculty of Commerce and Management \\ Eastern University \\ E-mail: antonarulrajah@yahoo.com \\ And \\ H.H.D.N.P. Opatha \\ Professor of HRM (on Merit) \\ Department of HRM \\ Faculty of Management Studies and Commerce \\ University of Sri Jayewardenepura \\ E-mail: hopatha@yahoo.com
}

\begin{abstract}
This study explores a gap in the empirical knowledge with regard to the personal qualities/characteristics expected by the organisations for key HRM jobs in the field of HRM in Sri Lanka. The research questions of this exploratory study were: What are the personal qualities/characteristics needed or expected by the organizations which are operating in Sri Lanka for key HRM jobs? And is it possible to develop a sort of illustration to understand personal qualities/characteristics expected for key HRM jobs in Sri Lanka? In order to answer the research questions systematically, an exploratory study was conducted by using eight key HRM job related employment advertisements appeared in the Sunday Observer newspaper for the period of 5 years (2006, 2007, 2008, 2009 and 2010). Time horizon of the study was longitudinal and HRM related job advertisement was the unit of analysis of the study.
\end{abstract}

Comprehensive and systematic content analysis along with descriptive statistics was applied to generate results and findings. The results of the study revealed that the personal qualities/characteristics are one of the very important requirements in selecting people for key HRM jobs in Sri Lanka. At the same time it differs from key job to another key job. When considering the personal qualities for the selected key HRM jobs, the findings revealed that there are 39 personal qualities related to the post of HR Director, 29 personal qualities related to the Group HR Manager, 41 personal qualities related to Head of HRM, 98 personal qualities related to the HR Manager, 81 personal qualities related to the HR Executive, 50 personal qualities related to the HR Officer, 32 personal qualities related to the Training Manager, and 15 personal qualities related to the post of Recruitment Manager.

With regard to the personal qualities eleven (11) key dimensions of personal qualities were identified in addition to the number of personal qualities including attitude related, personality related, good character related, team related, professional related, business related, people related, performance related, physical appearance related, leadership traits related and HR/HRM related aspects. The implications of these findings are useful to all 
stakeholders and professionals of HRM especially in developing good professionalism among current HR professionals in Sri Lanka and prospective personnel who wish to establish a career in HRM.

Key Words: Human Resource Management, Key HRM jobs, Personal Qualities

\section{Introduction}

In Sri Lanka, Human Resource Management (HRM) is becoming one of the most important functional fields in organizational management. Business organisations also have realized importance of HRM as a major factor in gaining a competitive advantage in a dynamic business environment. It has generally been observed that almost every large organization has a separate department for HRM under the leadership of HR Manager (HR Director or Group HR Manager in case of very large organizations and groups of companies). Establishment of separate academic departments for HRM in Sri Lankan universities including University of Sri Jayewardenepura, University of Kelaniya, and University of Colombo and introduction of HRM degree programs and specializations in HRM by Sri Lankan universities and technical colleges reflect increasing importance and consideration on HRM as an academic discipline in addition to practice. Like other fields such as Accounting and Engineering, HRM is being recognized as a prestigious and significant professional field.

An organization's performance directly depends on the individuals it employs. Strategies and decisions in staffing have a direct impact on whether the organization employs right or wrong individuals. Right individuals give right performance which leads to accomplish strategic needs of the organization. An organization cannot perform more than its employees perform. When done strategically, staffing can enable a company to acquire a sustainable competitive advantage that allows it to successfully fulfill its mission and reach its goals (Phillips and Gully, 2009). In this context HR staffing is critical for organizational success and it refers to establishing human resource management personnel in the human resource department of the organisation. It is indispensable to procure and employ people who possess right HRM knowledge, skills and special and general qualities to perform successfully jobs and positions of the human resource department. How effectively an organisation's human resources are managed depends in large part on the quality of the people in the human resource department (Schular and Youngblood, 1986). If the organisation has no right HR personnel, it is not in a position of performing HRM in right way and therefore it will not be able to achieve its goals. This paper has its focus on personal qualities required by Sri Lankan organizations from HR personnel or for key HRM jobs.

This study explores the personal qualities/characteristics expected by the organisations for key HRM jobs in Sri Lanka. Sri Lankan literature in connection with HRM field reveals that there is a material gap in the empirical knowledge regarding the above phenomenon. In order to fill this empirical knowledge gap, this paper addresses the following two specific research questions:

1. What are the personal qualities/characteristics needed or expected by the organizations which are operating in Sri Lanka for key HRM jobs? and

2. Is it possible to develop a sort of illustration to understand personal qualities expected for key HRM jobs in Sri Lanka? 


\section{The Research Objectives}

The objectives of this study were:

1. To explore the personal qualities/characteristics expected for the key HRM jobs; and

2. To develop an illustration of personal qualities/characteristics of key HRM jobs in Sri Lanka.

\section{Literature Review}

In the literature there were very few scholars who had mentioned about personal qualities/characteristics/attributes which are needed from the HRM professionals. In the view of Bergman (1992, as in Carrel et al. 1995), good judgment/common sense, flexible/resilient, leadership, action oriented, innovative thinking, and group dynamics are personal qualities required from HR Personnel. Kaufman (1994, as in Kleiman, 1997) stressed leadership qualities, managerial qualities and qualities which are related to HRM and business. In 1998, Sherman, Bohlander and Snell mentioned about four kinds of mastery such as business mastery, HR mastery, change mastery and personal credibility which were expected by the top executives from HR professionals. Sanghi (2004) has developed a checklist of HR competency audit. In that list she stressed needed personal attitudes and values of HR professionals in addition to their HR knowledge and HR skills. Deb (2006) grouped qualities of human resource professional/manager into two main groups. One is managerial qualities, and other one is professional qualities. Under the managerial qualities he pointed out seven specific qualities such as intelligence, conceptual clarity, analytical ability, motivational ability, emotional maturity, business acumen, and professional integrity. At the same time under the professional qualities he stated additional seven qualities such as empathy, communicator, human relation skill, knowledge, responsiveness, presentation, and management of change and culture.

In the Sri Lankan HRM literature, Opatha (2008 and 2009) pointed out seven competencies. In addition to the technical competence of HRM the human resource manager should possess competencies which include human relations competence (communicating, motivating, influencing people to change and leading), conceptual competence (planning, organising and controlling), intelligence (mental reasoning ability), appropriate personality (interest in people, appreciation in human capital, desire for developing people, pleasing appearance, adjustable and flexible, self- monitoring, extroversion to a certain extent, etc.), individual good character (moral excellence-ability to build and enhance virtues of responsibility, honesty, tolerance, patience, humility, etc. and minimise or eradicate vices of jealousy, greed, anger, stinginess, deception, corruption, retaliation etc.), and Personal/individual management competencies (ethical thinking, positive thinking, continuous learning, balancing life and work, time management, career management, and stress management). Out of these competences human relation competency, appropriate personality, intelligence and good character are highly related to personal qualities or characteristics of HR professional.

For the purpose of study, the term 'quality' or 'characteristic' is defined as a distinguishing feature or trait a person must possess to be perceived as a right HR professional. Indeed the concept is an abstract concept, and therefore it may be perceived alternatively as a competence. Qualities include attributes which are needed for a human resource professional to be successful on his/her job.

\section{Research Methodology}

In order to achieve the objectives of this research, an exploratory study was conducted by using all the HRM related employment advertisements appeared in the Sunday Observer 
newspaper for the period of 5 years (2006, 2007, 2008, 2009 and 2010). This study was longitudinal in time horizon and the unit of analysis of this study was HRM related job advertisement. The extent of the researchers' interference in this study was minimal as there had not been any manipulation and control of variables. As the study had been carried out in a natural environment where events occurred normally the study setting was non-contrived. Mainly by using comprehensive and systematic content analysis along with descriptive statistics, results and findings were generated.

\section{Results and Findings}

During the study period, a total of 3374 HRM job advertisements were collected from the organizations which are operating in Sri Lanka in all the sectors and out of which 1429 were related to the selected key HRM jobs which included Director-HR, Group Human Resource Manager, Head-HRM, Manager-HRM, HR Executive, HR Officer, Training Manager and Recruitment Manager. Results and findings were generated by exploring all the data in the light of research objectives.

Number of job advertisements and number of job vacancies relating to the advertisements for the selected 08 key jobs under study are given in Table 1. According to the Table, the highest number of job advertisements and vacancies were with the key job, HR Manager. HR Executive is the key job that had the next highest number of advertisements and vacancies. These two jobs become critical for a person who wants to excel in the HRM profession. Generally for a person who wishes to have an excellent career in the field of HRM, the job of HR Manager becomes a long term career goal and the job of HR Executive becomes a short term career goal. Tables from 2 to 9 provide expected personal qualities/characteristics for the eight key HRM jobs in Sri Lanka.

Table: 1 Key HRM Jobs: Number of Job Advertisements/Vacancies

\begin{tabular}{|l|c|c|c|c|c|c|}
\hline \multirow{2}{*}{ Selected Key HRM Jobs } & \multicolumn{5}{|c|}{ Year } & \multirow{2}{*}{ Total } \\
\cline { 2 - 6 } HR Director & $\mathbf{2 0 0 6}$ & $\mathbf{2 0 0 7}$ & $\mathbf{2 0 0 8}$ & $\mathbf{2 0 0 9}$ & $\mathbf{2 0 1 0}$ & \\
\hline No. of Job Advertisements & 07 & & & & & \\
\hline Number of Job Vacancies & 07 & 20 & 11 & 05 & 13 & $\mathbf{5 6}$ \\
\hline Group HR Manager & & & & & & 5 \\
\hline No. of Job Advertisements & 07 & 06 & 05 & 02 & 05 & $\mathbf{2 5}$ \\
\hline Number of Job Vacancies & 07 & 06 & 05 & 02 & 05 & $\mathbf{2 5}$ \\
\hline Head-HRM & & & & & & \\
\hline No. of Job Advertisements & 08 & 08 & 07 & 06 & 05 & $\mathbf{3 4}$ \\
\hline Number of Job Vacancies & 08 & 08 & 07 & 06 & 06 & $\mathbf{3 5}$ \\
\hline HR Manager & & & & & & \\
\hline No. of Job Advertisements & 116 & 139 & 130 & 63 & 114 & $\mathbf{5 6 2}$ \\
\hline Number of Job Vacancies & 122 & 147 & 160 & 68 & 121 & $\mathbf{6 1 8}$ \\
\hline HR Executive & & & & & & \\
\hline No. of Job Advertisements & 101 & 120 & 102 & 40 & 91 & $\mathbf{4 5 4}$ \\
\hline Number of Job Vacancies & 117 & 159 & 132 & 46 & 102 & $\mathbf{5 5 6}$ \\
\hline HR Officer & & & & & & \\
\hline No. of Job Advertisements & 27 & 35 & 28 & 27 & 41 & $\mathbf{1 5 8}$ \\
\hline Number of Job Vacancies & 35 & 36 & 30 & 53 & 44 & $\mathbf{1 9 8}$ \\
\hline Training Manager & & & & & & \\
\hline No. of Job Advertisements & 12 & 14 & 27 & 04 & 23 & $\mathbf{8 0}$ \\
\hline
\end{tabular}




\begin{tabular}{|l|c|c|c|c|c|c|}
\hline Number of Job Vacancies & 14 & 14 & 27 & 04 & 27 & $\mathbf{8 6}$ \\
\hline Recruitment Manager & & & & & & \\
\hline No. of Job Advertisements & 08 & 10 & 15 & 08 & 19 & $\mathbf{6 0}$ \\
\hline Number of Job Vacancies & 08 & 11 & 17 & 08 & 20 & $\mathbf{6 4}$ \\
\hline Note: Excluding Repetition
\end{tabular}

\section{Director- Human Resources Management}

Table: 2 Types of Personal Qualities/Characteristics and HR Director

\begin{tabular}{|c|c|c|c|c|c|}
\hline$\#$ & Qualities/Characteristics & Count & $\#$ & Qualities/Characteristics & Count \\
\hline 01 & A Real Team Player/Team Oriented & 23 & 21 & People Oriented & $\mathbf{0 3}$ \\
\hline 02 & Dynamic & 22 & 22 & Proactive & 03 \\
\hline 03 & High Level of Professionalism & 20 & 23 & A High Caliber & $\mathbf{0 2}$ \\
\hline 04 & Leadership qualities & 16 & 24 & Business Acumen & $\mathbf{0 2}$ \\
\hline 05 & Self-Motivated & 11 & 25 & Confidence & $\mathbf{0 2}$ \\
\hline 06 & Energetic & 10 & 26 & High Level of Enthusiasm & $\mathbf{0 2}$ \\
\hline 07 & Motivator & 09 & 27 & Passion & $\mathbf{0 2}$ \\
\hline 08 & Commitment & 08 & 28 & Sense of Humour & $\mathbf{0 2}$ \\
\hline 09 & Maturity and Emotional Balance & $\mathbf{0 5}$ & 29 & Service Oriented & $\mathbf{0 2}$ \\
\hline 10 & Resourceful & 05 & 30 & Talented & $\mathbf{0 2}$ \\
\hline 11 & Result Oriented & 05 & 31 & Willing to Put in Efforts Untiringly & $\mathbf{0 2}$ \\
\hline 12 & Challenging & 04 & 32 & Ambitious & 01 \\
\hline 13 & Drive & 04 & 33 & Creative & $\mathbf{0 1}$ \\
\hline 14 & Highly Qualified & 04 & 34 & Good Decision Maker & 01 \\
\hline 15 & Innovative & 04 & 35 & Hungry for Success & 01 \\
\hline 16 & Positive Attitude & 04 & 36 & Independent & 01 \\
\hline 17 & A Strong Intrinsic Motivation & $\mathbf{0 3}$ & 37 & Initiative & 01 \\
\hline 18 & Adaptable & $\mathbf{0 3}$ & 38 & Intellectual & 01 \\
\hline 19 & Flexible & $\mathbf{0 3}$ & 39 & Problem Solver & 01 \\
\hline 20 & Integrity & $\mathbf{0 3}$ & & & \\
\hline
\end{tabular}

\section{Group Human Resource Manager}

Table: 3 Types of Personal Qualities/Characteristics and GHRM

\begin{tabular}{|c|c|c|c|c|c|}
\hline \# & Qualities/Characteristics & Count & \# & Qualities/Characteristics & Count \\
\hline 01 & Dynamic & 10 & 16 & A Strong believer of open culture & 01 \\
\hline 02 & Results-oriented & 09 & 17 & A Strong Communicator & 01 \\
\hline 03 & Professionally qualified & 06 & 18 & Ambitious & 01 \\
\hline 04 & Team player & 06 & 19 & Committed to dead line & 01 \\
\hline 05 & Drive & 05 & 20 & Conceptual thinking & 01 \\
\hline 06 & Passionate & 04 & 21 & Focused & 01 \\
\hline 07 & A Strong Motivator & 03 & 22 & Forward Thinking & 01 \\
\hline 08 & Mature & $\mathbf{0 3}$ & 23 & Initiative & 01 \\
\hline 09 & Challenging & 02 & 24 & Negotiator & 01 \\
\hline 10 & Commitment & 02 & 25 & Organizer & 01 \\
\hline
\end{tabular}

An Exploratory Study on the Personal... 


\begin{tabular}{|l|l|l|l|l|l|}
\hline 11 & Confidence & $\mathbf{0 2}$ & 26 & Out-going & $\mathbf{0 1}$ \\
\hline 12 & Energetic & $\mathbf{0 2}$ & 27 & Proactive & $\mathbf{0 1}$ \\
\hline 13 & Self-Motivator & $\mathbf{0 2}$ & 28 & Resourcefulness & $\mathbf{0 1}$ \\
\hline 14 & Strategic Thinker & $\mathbf{0 2}$ & 29 & Young & $\mathbf{0 1}$ \\
\hline 15 & A self-starter & $\mathbf{0 1}$ & & & \\
\hline
\end{tabular}

\section{Head of Human Resource (Head-HRM)}

Table: 4 Types of Personal Qualities/Characteristics and Head-HRM

\begin{tabular}{|c|c|c|c|c|c|}
\hline \# & Qualities/Characteristics & Count & $\#$ & Qualities/Characteristics & Count \\
\hline 01 & Dynamic & 20 & 22 & Be Flexible & 01 \\
\hline 02 & Result Oriented & 10 & 23 & Business Acumen & 01 \\
\hline 03 & Self-Motivated & 10 & 24 & Commercially Astute Professional & $\mathbf{0 1}$ \\
\hline 04 & Team Player & 08 & 25 & Conscientious & 01 \\
\hline 05 & Professionalism & 05 & 26 & Diplomatic Approach & 01 \\
\hline 06 & Enthusiastic & 04 & 27 & Good Understanding & 01 \\
\hline 07 & Proactive & 04 & 28 & High Caliber & 01 \\
\hline 08 & Accept Challenges & $\mathbf{0 3}$ & 29 & Innovative & 01 \\
\hline 09 & Energetic & $\mathbf{0 3}$ & 30 & Leadership qualities & 01 \\
\hline 10 & Highly Committed & $\mathbf{0 3}$ & 31 & Loyalty & 01 \\
\hline 11 & A proven track records in the Field of HRM & $\mathbf{0 2}$ & 32 & Meticulous & 01 \\
\hline 12 & Effective & $\mathbf{0 2}$ & 33 & Nurture & 01 \\
\hline 13 & Integrity & 02 & 34 & Out-going Personality & 01 \\
\hline 14 & Optimistic & $\mathbf{0 2}$ & 35 & Self-Starter & 01 \\
\hline 15 & Passion for Achieving Excellence & $\mathbf{0 2}$ & 36 & Strive for Continuous Improvements at All Times & 01 \\
\hline 16 & Positive Attitudes & 02 & 37 & Talented & 01 \\
\hline 17 & Young Achiever & $\mathbf{0 2}$ & 38 & Tireless & 01 \\
\hline 18 & $\begin{array}{l}\text { A Natural Flair to engage with People at All } \\
\text { Levels }\end{array}$ & 01 & 39 & Uncompromising & 01 \\
\hline 19 & A Strong Generalized Background & 01 & 40 & Willingness to Work Outside Regular Hours & $\mathbf{0 1}$ \\
\hline 20 & All-Rounder & 01 & 41 & Work Independently & 01 \\
\hline 21 & Ambitious & 01 & & & \\
\hline
\end{tabular}

\section{Human Resource Manager (Manager-HRM)}

Table: 5 Types of Personal Qualities/Characteristics HR Manager

\begin{tabular}{|l|l|c|c|l|c|}
\hline$\#$ & Qualities/Characteristics & Count & $\#$ & Qualities/Characteristics & Count \\
\hline 01 & Dynamic & $\mathbf{1 4 1}$ & 50 & Self-Driven Personality & $\mathbf{0 6}$ \\
\hline 02 & Highly Professional & $\mathbf{1 0 7}$ & 51 & Self-Starter & $\mathbf{0 6}$ \\
\hline 03 & Strong Team Player/Team Spirit & $\mathbf{1 0 6}$ & 52 & An Abundance of Commonsense & $\mathbf{0 5}$ \\
\hline 04 & Self-Motivated & $\mathbf{7 7}$ & 53 & Diligent & $\mathbf{0 5}$ \\
\hline 05 & Results Oriented/Target Oriented & $\mathbf{7 3}$ & 54 & Honesty & $\mathbf{0 5}$ \\
\hline 06 & Suitably/Highly Qualified & $\mathbf{4 9}$ & 55 & Positive Demeanour & $\mathbf{0 5}$ \\
\hline 07 & Strong Leadership Qualities & $\mathbf{4 8}$ & 56 & Versatile & $\mathbf{0 5}$ \\
\hline 08 & Committed/unwavering & $\mathbf{4 2}$ & 57 & Working Independently & $\mathbf{0 5}$ \\
\hline
\end{tabular}

An Exploratory Study on the Personal... 


\begin{tabular}{|c|c|c|c|c|c|}
\hline & comr & & & & \\
\hline 09 & Prepared to Take Challenges & 41 & 58 & Good Organizer & 04 \\
\hline 10 & Energetic & 39 & 59 & Incessant Desire & 04 \\
\hline 11 & Strong/Highly Motivated/Motivator & 37 & 60 & $\begin{array}{l}\text { Ongoing Learning \& Self } \\
\text { Development }\end{array}$ & 04 \\
\hline 12 & Capable and Competent & 31 & 61 & Resourceful & 04 \\
\hline 13 & $\begin{array}{l}\text { Excellent Educational /Academic } \\
\text { Record }\end{array}$ & 30 & 62 & Strategic Thinker & 04 \\
\hline 14 & Proven/Sound Track Record & 26 & 63 & Trustworthiness & 04 \\
\hline 15 & Proactive & 22 & 64 & Empathetic & $\mathbf{0 3}$ \\
\hline 16 & Dedicated & 21 & 65 & Good Sense of Judgment & $\mathbf{0 3}$ \\
\hline 17 & Innovative & 21 & 66 & Good Understanding & $\mathbf{0 3}$ \\
\hline 18 & Self-Confidence & 21 & 67 & Highly Multi-Disciplined & 03 \\
\hline 19 & Young & 21 & 68 & Inspiration & $\mathbf{0 3}$ \\
\hline 20 & A Positive Attitude/Mindset & 20 & 69 & Intellect & 03 \\
\hline 21 & Highly/ Best Talented & 19 & 70 & Loyalty & 03 \\
\hline 22 & Initiative & 19 & 71 & Practical & $\mathbf{0 3}$ \\
\hline 23 & Ambitious Individual & 17 & 72 & Service Oriented & 03 \\
\hline 24 & Passion & 17 & 73 & An Individual of Good Character & $\mathbf{0 2}$ \\
\hline 25 & Smart and physical stamina & 17 & 74 & Diplomatic & 02 \\
\hline 26 & Strong/Sturdy Personality & 17 & 75 & Ethical Commitment & $\mathbf{0 2}$ \\
\hline 27 & High Caliber & 15 & 76 & Forceful Player & 02 \\
\hline 28 & Relentless Drive/Drive & 15 & 77 & Foresight Qualities & 02 \\
\hline 29 & Strive for Achievement & 14 & 78 & Forward Thinking & 02 \\
\hline 30 & Matured & 13 & 79 & Goal Oriented & $\mathbf{0 2}$ \\
\hline 31 & Work Under Pressure & 12 & 80 & Go-Getter Attitude & $\mathbf{0 2}$ \\
\hline 32 & Accept Responsibilities & 12 & 81 & Out of Box Professional Attitudes & 02 \\
\hline 33 & Be Flexible & 11 & 82 & Strong Attitude & $\mathbf{0 2}$ \\
\hline 34 & Excellent & 11 & 83 & Willing to Work Long Hours & $\mathbf{0 2}$ \\
\hline 35 & High Sense of Enthusiasm & 11 & 84 & Balanced Approach & 01 \\
\hline 36 & Integrity & 11 & 85 & Best in the Field & 01 \\
\hline 37 & Pleasing Personality & 11 & 86 & Can do Attitude & 01 \\
\hline 38 & Outgoing Personality & 10 & 87 & Commercially minded & 01 \\
\hline 39 & Communicator & 09 & 88 & Disciplined & 01 \\
\hline 40 & Confidentiality & 09 & 89 & Flare for Public Relations & 01 \\
\hline 41 & $\begin{array}{l}\text { Market Intelligence/Business } \\
\text { Acumen }\end{array}$ & 08 & 90 & Focused Approach & 01 \\
\hline 42 & Meaningful Contributor & $\mathbf{0 8}$ & 91 & Good Physical condition & 01 \\
\hline 43 & Outstanding/Exceptional & 08 & 92 & Humility & 01 \\
\hline 44 & People Oriented/People Friendly & 07 & 93 & Look ahead & 01 \\
\hline 45 & Career Minded & 06 & 94 & Meticulous & 01 \\
\hline 46 & Creative & 06 & 95 & Open Minded & 01 \\
\hline 47 & Determination & 06 & 96 & Resilient & 01 \\
\hline 48 & Hardworking & 06 & 97 & Sincerity & 01 \\
\hline 49 & Independent Thinker & 06 & 98 & Virtue & 01 \\
\hline
\end{tabular}




\section{Human Resource Executive (HR Executive)}

Table: 6 Types of Personal Qualities/Characteristics and HR Executive

\begin{tabular}{|c|c|c|c|c|c|}
\hline$\#$ & Qualities/Characteristics & Count & \# & Qualities/Characteristics & Count \\
\hline 01 & Dynamic & 141 & 42 & Honesty & 05 \\
\hline 02 & Team Player/Oriented/Team Spirit & 102 & 43 & Confidentiality & 04 \\
\hline 03 & Self Motivated & 70 & 44 & $\begin{array}{l}\text { Continuous Improvement All the } \\
\text { Time }\end{array}$ & 04 \\
\hline 04 & Young & 56 & 45 & Ethical Minded & 04 \\
\hline 05 & Professionally Qualified & 55 & 46 & Excellent & 04 \\
\hline 06 & Results /Target Oriented & 54 & 47 & Good Understanding & 04 \\
\hline 07 & Energetic & 41 & 48 & Methodical, Systematic \& Organised & 04 \\
\hline 08 & Accepting Challenges & 32 & 49 & People Oriented & 04 \\
\hline 09 & Committed & 32 & 50 & Vibrant & 04 \\
\hline 10 & Enthusiasm & 28 & 51 & $\begin{array}{l}\text { Willing to Work Highly Competitive } \\
\text { Environment }\end{array}$ & 04 \\
\hline 11 & Leadership Qualities & 27 & 52 & Analytical Mind & 03 \\
\hline 12 & Dedicated & 25 & 53 & Communicator & 03 \\
\hline 13 & Highly Motivated & 19 & 54 & Contributor & $\mathbf{0 3}$ \\
\hline 14 & Drive/Self Driven & 18 & 55 & Diplomatic & $\mathbf{0 3}$ \\
\hline 15 & Highly Talented & 18 & 56 & Exceptional & $\mathbf{0 3}$ \\
\hline 16 & Positive Minded/Attitudes & 18 & 57 & Positive Personality & 03 \\
\hline 17 & Right Academic Background & 18 & 58 & Proper Attitude for Work & 03 \\
\hline 18 & Initiative & 17 & 59 & Quick \& Willing Learner & 03 \\
\hline 19 & Suitably Qualified & 16 & 60 & Self Managed & 03 \\
\hline 20 & Achievement Oriented & 15 & 61 & All-rounder & 02 \\
\hline 21 & Innovative & 15 & 62 & Client Pleaser & 02 \\
\hline 22 & High Caliber & 12 & 63 & Development Oriented & 02 \\
\hline 23 & Passion & 12 & 64 & Empathy & 02 \\
\hline 24 & Proactive & 12 & 65 & Flexible & 02 \\
\hline 25 & Smart & 12 & 66 & Inspiration & 02 \\
\hline 26 & Confident/Self-Confident & 11 & 67 & Look Forward & 02 \\
\hline 27 & Competent & 10 & 68 & Personal Discipline & 02 \\
\hline 28 & Hard-Working & 10 & 69 & Positive Interpersonal Relations & 02 \\
\hline 29 & Outgoing Personality/Person & 10 & 70 & $\begin{array}{l}\text { Proven Record in Extra Curricular } \\
\text { Activities }\end{array}$ & 02 \\
\hline 30 & Responsible & $\mathbf{0 8}$ & 71 & Strategic Thinkers & 02 \\
\hline 31 & Best Attitudes & 07 & 72 & Assertive & 01 \\
\hline 32 & Capable & 07 & 73 & Career Minded & 01 \\
\hline 33 & Career Oriented/Minded & 06 & 74 & Creative & 01 \\
\hline 34 & Go Getters Attitude & 06 & 75 & Customer Orientation & 01 \\
\hline 35 & Integrity & 06 & 76 & Dare to Do Spirit & 01 \\
\hline 36 & Intelligent & 06 & 77 & Determination & 01 \\
\hline 37 & Mature & 06 & 78 & Emotional Resilience & 01 \\
\hline 38 & Pleasing Personality & 06 & 79 & Have a Can Do Attitudes & 01 \\
\hline 39 & Willing to Work Long Hours & 06 & 80 & Self-starter & 01 \\
\hline
\end{tabular}

An Exploratory Study on the Personal... 


\begin{tabular}{|l|l|l|l|l|l|}
40 & Working Independently & $\mathbf{0 6}$ & 81 & Swift & $\mathbf{0 1}$ \\
\hline 41 & Ambitious & $\mathbf{0 5}$ & & & \\
\hline
\end{tabular}

\section{Human Resource Officer (HR Officer)}

Table: 7 Types of Personal Qualities/Characteristics and HR Officer

\begin{tabular}{|c|c|c|c|c|c|}
\hline$\#$ & Qualities/Characteristics & Count & $\#$ & Qualities/Characteristics & Count \\
\hline 01 & Team Player/Oriented/Team Spirit & 27 & 26 & Initiative & $\mathbf{0 3}$ \\
\hline 02 & Dynamic & 30 & 27 & Intelligent & 03 \\
\hline 03 & Highly Qualified & 20 & 28 & Proactive & $\mathbf{0 3}$ \\
\hline 04 & Self Motivated & 17 & 29 & Diplomacy & $\mathbf{0 2}$ \\
\hline 05 & Results Oriented & 13 & 30 & Efficient & $\mathbf{0 2}$ \\
\hline 06 & Work with minimum supervision & 11 & 31 & Highly Multidisciplined & $\mathbf{0 2}$ \\
\hline 07 & Energetic & 10 & 32 & Leadership Qualities & 02 \\
\hline 08 & Committed & 09 & 33 & Willing to Help & 02 \\
\hline 09 & Flexible & 08 & 34 & Work Independently & 02 \\
\hline 10 & Innovative & 08 & 35 & Career Oriented/Minded & 01 \\
\hline 11 & Young & 08 & 36 & Challenge Taker & 01 \\
\hline 12 & Mature Person & 07 & 37 & Confident & 01 \\
\hline 13 & Ambitious & 06 & 38 & Confidentiality & 01 \\
\hline 14 & Creative & 06 & 39 & Enthusiastic & 01 \\
\hline 15 & Work under Pressure & 06 & 40 & Go-getter & 01 \\
\hline 16 & Capable and competent & 05 & 41 & Integrity & 01 \\
\hline 17 & Dedicated & 05 & 42 & Passion & 01 \\
\hline 18 & Higher Caliber & 05 & 43 & Patient & 01 \\
\hline 19 & Highly Motivated & 05 & 44 & Positive Attitude & 01 \\
\hline 20 & Friendly and People Oriented & 04 & 45 & Reliable & 01 \\
\hline 21 & In Response to Changing Needs & 04 & 46 & Self Managed & 01 \\
\hline 22 & Pleasing Personality & 04 & 47 & Sense of Self Achievement & 01 \\
\hline 23 & Tact & 04 & 48 & Sense of Self Development & 01 \\
\hline 24 & Drive/Self Driven & 03 & 49 & Trustworthy & 01 \\
\hline 25 & Honest & 03 & 50 & Unencumbered & 01 \\
\hline
\end{tabular}

\section{Training Manager}

Table: 8 Types of Personal Qualities/Characteristics/Attributes and Training Manager

\begin{tabular}{|l|l|c|c|l|c|}
\hline$\#$ & Qualities/Characteristics & Count & $\#$ & Qualities/Characteristics & Count \\
\hline 01 & Dynamic & $\mathbf{2 6}$ & 17 & Creative & $\mathbf{0 2}$ \\
\hline 02 & Highly Motivated & $\mathbf{1 3}$ & 18 & Dedicated & $\mathbf{0 2}$ \\
\hline 03 & Results Oriented & $\mathbf{1 0}$ & 19 & High Caliber & $\mathbf{0 2}$ \\
\hline 04 & Self Motivated & $\mathbf{0 8}$ & 20 & Initiative & $\mathbf{0 2}$ \\
\hline 05 & Team Player/Oriented/Team Spirit & $\mathbf{0 8}$ & 21 & Capable & $\mathbf{0 1}$ \\
\hline 06 & Innovative & $\mathbf{0 7}$ & 22 & Committed & $\mathbf{0 1}$ \\
\hline 07 & Talented & $\mathbf{0 6}$ & 23 & Focused & $\mathbf{0 1}$ \\
\hline 08 & Professionally qualified & $\mathbf{0 5}$ & 24 & Good Leader & $\mathbf{0 1}$ \\
\hline
\end{tabular}




\begin{tabular}{|l|l|l|l|l|l|}
09 & Sober and Mature Person & $\mathbf{0 5}$ & 25 & Hard working & $\mathbf{0 1}$ \\
\hline 10 & Drive/Self Driven & $\mathbf{0 4}$ & 26 & Passion & $\mathbf{0 1}$ \\
\hline 11 & Excellent & $\mathbf{0 4}$ & 27 & People Oriented & $\mathbf{0 1}$ \\
\hline 12 & Proactive & $\mathbf{0 4}$ & 28 & Physically Fit & $\mathbf{0 1}$ \\
\hline 13 & Suitably Qualified & $\mathbf{0 4}$ & 29 & Service Oriented & $\mathbf{0 1}$ \\
\hline 14 & Energetic & $\mathbf{0 3}$ & 30 & Strictly Disciplinarian & $\mathbf{0 1}$ \\
\hline 15 & Self-Starter & $\mathbf{0 3}$ & 31 & Strong Personality & $\mathbf{0 1}$ \\
\hline 16 & Change Agent & $\mathbf{0 2}$ & 32 & Trustworthy Person & $\mathbf{0 1}$ \\
\hline
\end{tabular}

\section{Recruitment Manager}

Table: 9 Types of Personal Qualities/Characteristics/Attributes and Recruitment Manager

\begin{tabular}{|l|l|c|c|l|c|}
\hline$\#$ & Qualities/Characteristics & Count & $\#$ & Qualities/Characteristics & Count \\
\hline 01 & Dynamic & $\mathbf{1 0}$ & 09 & $\begin{array}{l}\text { Good understanding of human } \\
\text { relations }\end{array}$ & $\mathbf{0 2}$ \\
\hline 02 & Team player & $\mathbf{0 6}$ & 10 & Outgoing personality & $\mathbf{0 2}$ \\
\hline 03 & Energetic individual & $\mathbf{0 5}$ & 11 & Well/Highly organized & $\mathbf{0 2}$ \\
\hline 04 & Self-motivated & $\mathbf{0 4}$ & 12 & Empathetic & $\mathbf{0 1}$ \\
\hline 05 & Talented & $\mathbf{0 4}$ & 13 & Positive Mined & $\mathbf{0 1}$ \\
\hline 06 & High Caliber & $\mathbf{0 3}$ & 14 & Proactive & $\mathbf{0 1}$ \\
\hline 07 & Professionally qualified & $\mathbf{0 3}$ & 15 & Trustworthy Personality & $\mathbf{0 1}$ \\
\hline 08 & Good Communicator & $\mathbf{0 2}$ & & & \\
\hline
\end{tabular}

Discussion

Full list of personal qualities/characteristics expected by the Sri Lankan organizations from HR professionals was presented under the results and findings. The list contains almost each item in terms of quality that had been required by the organizations under the study. In this context two types of discussions were attempted. First one is identification of top five personal qualities/characteristics expected by the organizations regarding each HRM job and second one is overall discussion with regard to certain types or key categories of key personal qualities/characteristics expected by the organizations. The following Exhibit and Figure 1 relate to the first attempt and Figure 2 and the Appendix relate to the second attempt.

Exhibit: 1 Top Five Personal Qualities/Characteristics expected by the Organizations Regarding each HRM Job

\begin{tabular}{|c|l|c|l|}
\hline$\#$ & \multicolumn{1}{|c|}{ HRM Job } & $\#$ & \multicolumn{1}{|c|}{ Top Five personal qualities/characteristics/attributes } \\
\hline 01 & Director-HR/HRM & 01 & A Real Team Player/Team Oriented \\
\hline \multirow{4}{*}{} & Number of content identified - 39 & 02 & Dynamic \\
\cline { 3 - 4 } & & 03 & High Level of Professionalism \\
\cline { 3 - 4 } & & 04 & Leadership qualities \\
\cline { 3 - 4 } & 05 & Self-Motivated \\
\hline \multirow{2}{*}{$\mathbf{0 2}$} & Group HR Manager & & \\
\cline { 3 - 4 } & Number of content identified $\mathbf{- 2 9}$ & 01 & Dynamic \\
\hline & & 02 & Results Oriented \\
\cline { 3 - 4 } & & 03 & Professionally Qualified \\
\cline { 3 - 4 } & & 05 & Team player \\
\hline \multirow{2}{*}{$\mathbf{0 3}$} & Head of HR & 01 & Drive \\
\hline & Number of content identified $\mathbf{- 4 1}$ & 02 & Results Oriented \\
\hline
\end{tabular}

An Exploratory Study on the Personal... 


\begin{tabular}{|c|c|c|c|}
\hline & & 03 & Self-Motivated \\
\hline & & 04 & Team Player \\
\hline & & 05 & Professionalism \\
\hline \multirow[t]{5}{*}{04} & HR Manager & 01 & Dynamic \\
\hline & Number of content identified -98 & 02 & Highly professional \\
\hline & & 03 & Strong Team Player/Team Spirit \\
\hline & & 04 & Self-Motivated \\
\hline & & 05 & Results Oriented/Target Oriented \\
\hline \multirow[t]{5}{*}{05} & HR Executive & 01 & Dynamic \\
\hline & Number of content identified -81 & 02 & Team Player/Oriented/Team Spirit \\
\hline & & 03 & Self Motivated \\
\hline & & 04 & Young \\
\hline & & 05 & Professionally Qualified \\
\hline \multirow[t]{5}{*}{06} & HR Officer & 01 & Team Player/ Oriented/Team Spirit \\
\hline & Number of content identified -50 & 02 & Dynamic \\
\hline & & 03 & Highly Qualified \\
\hline & & 04 & Self Motivated \\
\hline & & 05 & Results Oriented \\
\hline \multirow[t]{5}{*}{07} & Training Manager & 01 & Dynamic \\
\hline & Number of content identified -32 & 02 & Highly Motivated \\
\hline & & 03 & Results Oriented \\
\hline & & 04 & Self Motivated \\
\hline & & 05 & Team Player/ Team Player/Oriented/Team Spirit \\
\hline \multirow[t]{5}{*}{08} & Recruitment Manager & 01 & Dynamic \\
\hline & Number of content identified -15 & 02 & Team player \\
\hline & & 03 & Energetic individual \\
\hline & & 04 & Self motivated \\
\hline & & 05 & Talented \\
\hline
\end{tabular}

Here, it is possible to understand that there were very much similarities regarding certain personal qualities of selected HRM jobs. Dynamic, team player/oriented/team spirit, motivated/self-motivated, and results oriented were commonly and very frequently stated /expected four personal qualities by the organizations irrespective of different HRM jobs in Sri Lanka. The label 'common personal qualities' could be given to these four identified qualities.

Dynamic means unstable, changing, not being static. This quality is considered as one of the most important common qualities for all the key HRM jobs. It may be due to the reason that a human resource professional has to face various different situations which require new way of thinking and thinking out of the box rather than limiting to one framework of looking at problems, and being closed minded with pre-determined conclusions. Team orientation means state of being directed as a team. Human resource professional (specially head of HR department) has to work as a member of the team that consists of other heads (line and staff) who are committed to a common purpose, i.e. achieving strategic needs (vision, mission and strategic goals) of the organization. In addition HR professionals are supposed to coordinate HR related works with other managers in the organization and they have to team up themselves within the HR department. 
Self-motivated means to generate a force that causes the person (in this context HR professional) to engage in a particular behavior (in this context to perform relevant HR duties and responsibilities) rather than other behaviors. HR professional is supposed to have decision of performing relevant duties and responsibilities seriously; decision of putting much effort into job performance; and decision of spending hours and hours to keep working at job performance. Results oriented means state of being directed as a person of results rather than a person of traits and behaviors. One may utilize his/her traits and engage in right behaviors but may not produce right outputs/results (mainly quality and quantity of work). As results are the most important at the final analysis of achieving strategic needs of the organization, HR professionals are supposed to produce right or expected results which will create value to realization of strategic goals of the organization. Figure 1 shows linkage between common personal qualities and key HRM jobs.

Figure: 1 Linkage between Common Personal Qualities and Key HRM Jobs

\begin{tabular}{|l|l|l|}
\hline Being Dynamic & 1. & Director-HR \\
2. & Group HR Manager \\
3. & Head of HR \\
Self-Motivated Orientation & 4. & HR Manager \\
5esult-Oriented & 5. & HR Executive \\
& 6. & HR Officer \\
7. & Training Manager \\
8. & Recruitment \\
& & Manager \\
\hline
\end{tabular}

Figure: 2 Key Categories of Personal Qualities/Characteristics expected by the Organizations to Perform HRM Jobs in Sri Lanka

\begin{tabular}{|c|c|c|}
\hline $\begin{array}{c}\text { Attitude } \\
\text { Component }\end{array}$ & $\begin{array}{c}\text { Personality } \\
\text { Component }\end{array}$ & $\begin{array}{c}\text { Good Character (Virtue) } \\
\text { Component }\end{array}$ \\
\hline $\begin{array}{c}\text { Team } \\
\text { Component }\end{array}$ & $\begin{array}{c}\text { Professional } \\
\text { Component }\end{array}$ & $\begin{array}{c}\text { Business } \\
\text { Component }\end{array}$ \\
\hline $\begin{array}{c}\text { People } \\
\text { Component }\end{array}$ & $\begin{array}{c}\text { Key Categories of } \\
\text { Personal Qualities } \\
\text { Expected by the } \\
\text { Organizations to } \\
\text { Perform HRM Jobs }\end{array}$ & $\begin{array}{c}\text { Performance } \\
\text { Component }\end{array}$ \\
\hline $\begin{array}{c}\text { Physical Character related } \\
\text { Component }\end{array}$ & $\begin{array}{c}\text { Leadership } \\
\text { Traits }\end{array}$ & $\begin{array}{c}\text { HR/HRM related } \\
\text { Component }\end{array}$ \\
\hline
\end{tabular}

The above Figure was developed after investigating 176 (after eliminating overlapping) personal qualities/characteristics identified from this exploratory study. Regarding personal qualities/characteristics of HRM professionals Bergman (1992), Kaufman (1994), Sherman et 
al (1998), Sanghi (2004), Deb (2006) and Opatha (2007 and 2009) have emphasized that many types of personal qualities/characteristics are necessary for HRM professionals. Individually 176 (overall/summary) personal qualities/characteristics were identified from the findings of this study and further eleven (11) key categories of personal qualities needed to perform HRM jobs were identified from those 176 individual personal qualities through classification. In general, results of this study were almost similar with observations of the above mentioned authors and however this study provides a more comprehensive view about personal qualities/characteristics needed for HR professionals, especially in the Sri Lankan context.

\section{Conclusion}

Content analysis of the relevant 1429 newspaper recruitment advertisements revealed 176 personal qualities expected by Sri Lankan organizations for 08 key HRM jobs. There are 39 personal qualities related to the post of HR Director, 29 personal qualities related to the Group HR Manager, 41 personal qualities related to Head of HRM, 98 personal qualities related to the HR Manager, 81 personal qualities related to the HR Executive, 50 personal qualities related to the HR Officer, 32 personal qualities related to the Training Manager, and 15 personal qualities related to the post of Recruitment Manager. The most important five personal qualities for each key HRM job have been presented. Further investigation resulted in four common personal qualities which are highly demanded by the organizations under study and they include being dynamic, team orientation, self-motivated and result-oriented. 11 key categories of personal qualities were identified through classification and they include attitude, personality, good character (virtue), team, professional, business, people, performance, physical character, leadership, and HRM related component.

Results of the study have direct implications on all stakeholders and HRM professionals in developing good professionalism among current HR professionals in Sri Lanka and prospective personnel who wish to establish a career in HRM. Senior university teachers need to take into account of these results in order to develop a good curriculum of HRM and teachers and trainers who are directly involved in teaching and training individuals today to make them future successful professionals in HRM should develop learning materials and applications (cases, critical incidents, skill builders etc.) relating to the identified key categories and common personal qualities. Those who currently seek employment opportunities in the field of HRM need to get prepared for enhancing those personal qualities so as to increase the likelihood of getting selected for the relevant job vacancies.

The data used in this study were secondary data and therefore avenues for further investigation by collecting primary data are possible. Another interesting avenue for future research is to carry out similar studies in other fields of business management including marketing, finance, operation, business information technology etc.

\section{References}

Bergman, T. (1992), "Preparing to Enter and Succeed in Human Resource Management," SAM Advanced Management Journal (Winter 1992):37. [Carrell, M. R, Elbert, N.F and Hatfield, R.D (1995), HRM Global Strategies for Managing a Diverse Workforce, $5^{\text {th }}$ ed, New Jersey: Prentice Hall].

Deb, T. (2006), Strategic Approach to Human Resource Management, Concept, Tools and Application, New Delhi: Atlantic Publishers \& Distributors. 
Kaufman, B.E. (1994), What companies want from HR graduates, HRMagazine, September, 84-86. [Kleiman, L. S (1997), Human Resource Management: A Tool for Competitive Advantage, New York: West Publishing Company].

Opatha, H.H.D.N.P. (2007), Competencies as Human Assets: A Perspective, Sri Lankan Journal of Human Resource Management, Vol. 2, No.1, pp.1-13.

Opatha, H.H.D.N.P. (2009), Human Resource Management: Personnel, Colombo: Department of HRM, University of Jayewardenepura.

Phillips, J.M. and Gully, S.M. (2009), Strategic Staffing, New York: Pearson Prentice Hall.

Sanghi, S. (2004), The Handbook of Competency Mapping, Understanding, Designing and Implementing Competency Models in Organizations, New Delhi: Response Books (A division of Sage Publications India Pvt Ltd).

Schuler, R.S. and Youngblood, S.A. (1986), Effective Personnel Management $2^{\text {nd }}$ ed., New York: West Publishing Company.

Sherman, A., Bohlander, G. and Snell, S. (1998), Managing Human Resources, $11^{\text {th }}$ ed, Cincinnati, Ohio: South-Western College Publishing.

The Appendix: Types of Personal Qualities/Characteristics expected/needed to perform HRM Jobs

\# Types of Personal Qualities/Characteristics

01. Attitude Related

1.1 Positive Attitude/Mindset

1.2 Best Attitudes

1.3 Dare to Do Spirit

1.4 Go-Getter Attitude

1.5 Have a Can Do Attitude

1.6 Open Minded

1.7 Optimistic

1.8 Out of Box Professional Attitudes

1.9 Proper Attitude for Work

1.10 Strong Attitude

02. Personality Related

2.1 Outgoing Personality

2.2 Trustworthy Personality

2.3 Strong Personality

2.4 Self-Driven Personality

2.5 Sturdy Personality

2.6 Pleasing Personality

2.7 Positive Personality

2.8 Pleasant Personality

2.9 Assertive

03. Good Character (Virtue) Related

3.1 An Individual of Good Character

3.2 Good Sense of Judgment

3.3 Honesty/ Honest

3.4 Humility

3.5 Loyalty

3.6 Sincerity

3.7 Disciplined

3.8 Reliable

3.9 Patient

An Exploratory Study on the Personal... 
04. Team Related

4.1 A Real Team Player

4.2 Team Oriented

4.3 Team Sprit

05. Professional Related

5.1 Career Oriented/Minded

5.2 Confidentiality

5.3 High Level of Professionalism/ Professionalism

5.4 Professional Approach

5.5 Professionally qualified

\section{Business Related}

6.1 Client Pleaser

6.2 Commercially Astute Professional

6.3 Commercially minded

6.4 Customer Oriented

6.5 Market Intelligence

6.6 Service Oriented

\section{People Related}

7.1 A Natural Flair to engage with People at All Levels

7.2 An Abundance of Commonsense

7.3 Diplomatic Approach/ Diplomacy/ Diplomatic

7.4 Empathy/ Empathetic

7.5 Flare for Public Relations

7.6 Flexible

7.7 Friendly/ People Friendly

7.8 Good communicator

7.9 Good understanding of human relations

7.10 Nurture

7.11 People Oriented

7.12 Positive Interpersonal Relations

7.13 Sense of Humour

7.14 Tact

7.15 Willing to Help

\section{Performance Related}

8.1 A Strong believer of open culture

8.2 Achiever/ Achievement Oriented

8.3 All-Rounder

8.4 Capable

8.5 Commitment/ Highly Committed/ Committed/unwavering commitment

8.6 Committed to dead line

8.7 Competent

8.8 Conscientious

8.9 Diligent

8.10 Effective

8.11 Efficient

8.12 Excellent

8.13 Excellent Educational /Academic Record

8.14 Exceptional

8.15 Goal Oriented 


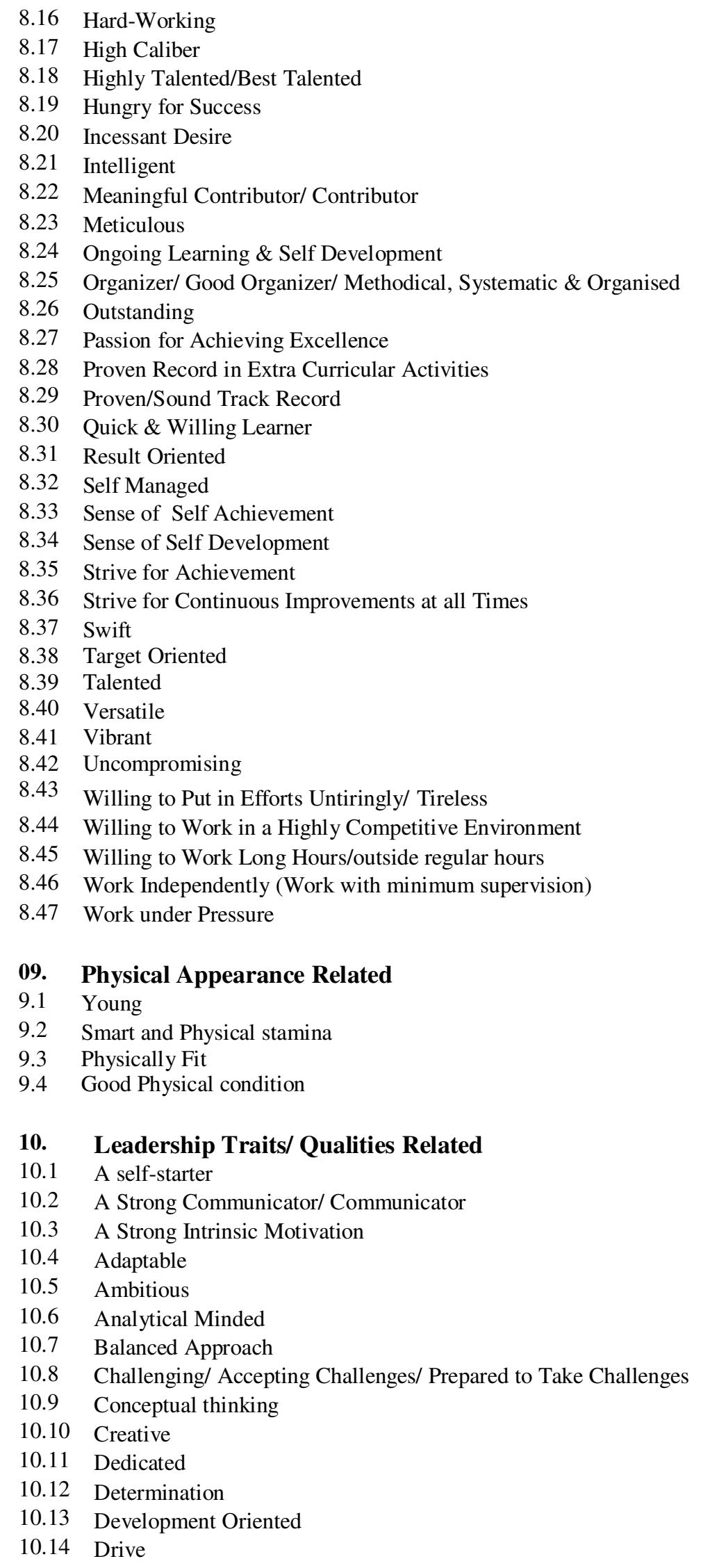




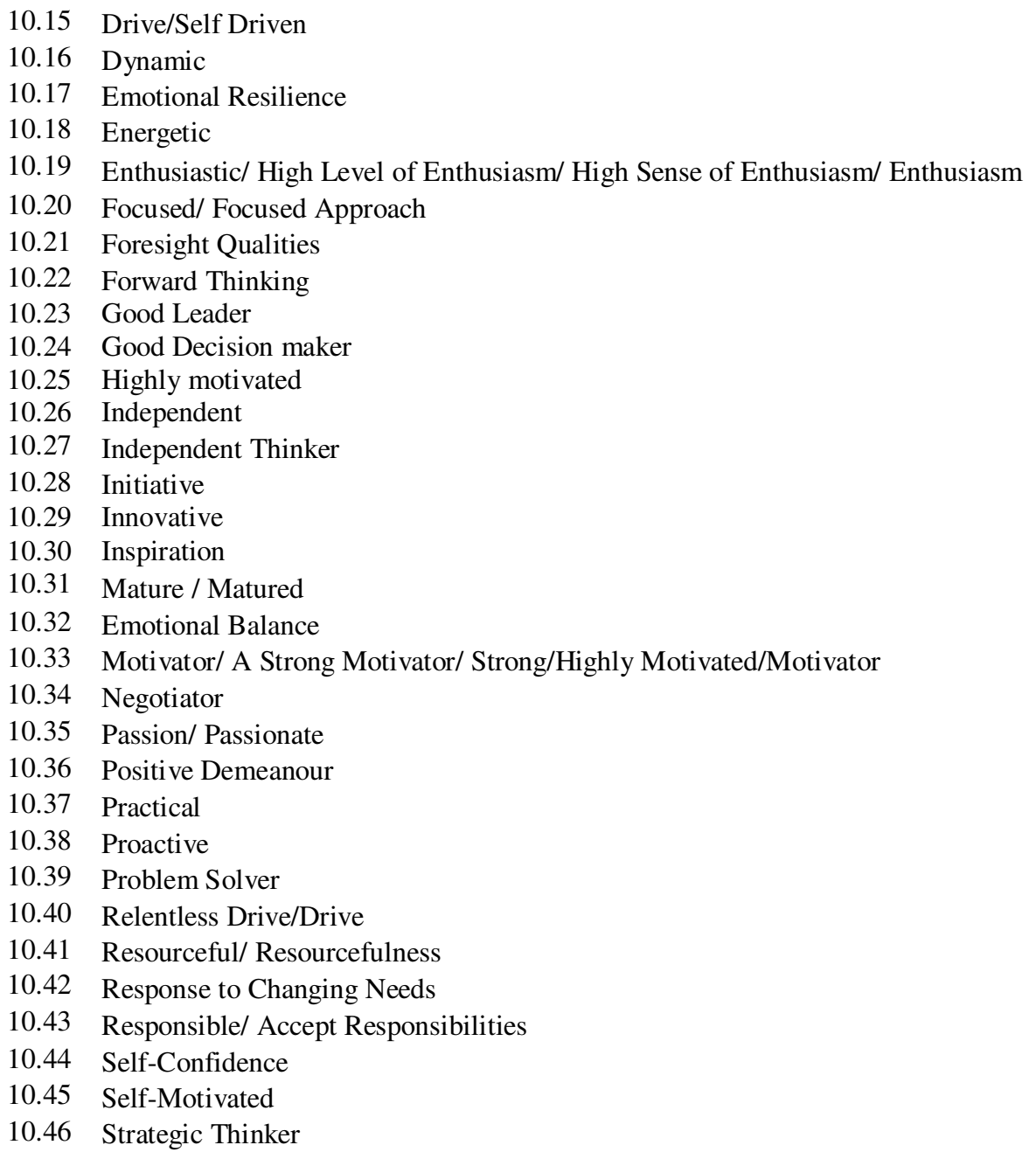

\section{HRM Related}

11.1 A proven track records in the Field of HRM

11.2 A Strong Generalized HRM Background

11.3 One of the Best in the Field

11.4 Forceful Player in the Field

11.5 Right Academic Background (HRM)

11.6 Suitably Qualified (HRM) 